\title{
Naturkundliche Impressionen aus Peru (1. Teil) - Die Umgebung von Oxapampa, insbesondere der Nationalpark Yanachaga Chemillén
}

\author{
Günter Gerlach
}

\begin{abstract}
In terms of area, Peru is the third largest country in South America after Brazil and Argentina, and due to its proximity to the equator it has a largely tropical climate. Its geography is highly structured by the Andes and the Amazonian lowlands. Therefore, it has a multitude of ecological niches in which an extremely diverse flora and fauna have developed. Due to a long-term stay, the author was able to conduct extensive natural history studies in different areas and altitudes and thus in different climates. These are presented in case studies in a loose sequence, with a focus on the fascinating pollination biology of plants. For research reasons, only humid habitats from different departments of Peru are presented here.
\end{abstract}

\section{Zusammenfassung}

Peru ist flächenmäßig nach Brasilien und Argentinien das drittgrößte Land in Südamerika, durch seine Äquatornähe beherbergt es weitgehend tropische Klimata. Durch die Anden und das Amazonische Tiefland ist es geografisch reich gegliedert. So weist es eine Vielzahl ökologischer Nischen auf, in denen sich eine extrem diverse Flora und Fauna entwickelt hat. Durch einen Langzeitaufenthalt war es dem Autor möglich, ausgiebige naturkundliche Studien in verschiedenen Gebieten unterschiedlicher Höhenlagen und somit unterschiedlichstem Klima zu betreiben. Diese werden in Fallbeispielen in lockerer Reihung nacheinander vorgestellt, wobei die faszinierende Bestäubungsbiologie der Pflanzen einen Schwerpunkt bildet. Forschungsbedingt werden hier nur humide Biotope aus verschiedenen Departements Perus vorgestellt.

\section{Vorwort}

Während eines zum Teil wegen der Covid-19-Pandemie unfreiwilligen Langzeitaufenthalts in Peru hatte der Autor die Möglichkeit, eine Forschungsgenehmigung im Nationalpark Yanachaga Chemillén zu erlangen. Ermutigt durch Rocío Rojas und Rodolfo VÁsquez, den Begründern und Leitern des Jardín Botánico de Missouri (eine eigenständige Institution, die nur angelehnt an den Missouri Botanical Garden agiert) beantragte der Autor diese Genehmigung, die auch problemlos erteilt wurde. Rocío Rojas und Rodolfo VÁsQuez gilt großer Dank für die Ermutigung und Begleitung auch im unwegsamen Gelände. Der Nationalpark war nicht einfach zu erreichen, obwohl er in Sichtweite der Unterkunft des Autors in Oxapampa lag. Er umfasst die Rand-Kordillere der Anden im Bereich von Zentralperu. Ostwärts vom amazonischen Tiefland abgeschirmt, zunächst durch das Vorgebirge des Sira und dann von der niedrigen Bergkette der Selvas de San Matias, erhebt sich die Kordillere Yanachaga von $300 \mathrm{~m}$ bis über $4600 \mathrm{~m}$. Sie wurde 1986 auf Betreiben des damaligen Umweltministers AnToNIO BRACK EGG weitläufig unter Schutz gestellt.

Der U.S.-amerikanische Botaniker RoBiN Foster berichtete von einer Wanderung mit AN-
TONio Brack EgG: „Ahh, Oxapampa. Wie ich diesen Ort geliebt habe, zumindest bevor er aufgebaut wurde. Ich betrachtete ihn sogar als einen Ort, um mich zur Ruhe zu setzen. Mich faszinierte vor allem meine Übernachtungswanderung mit Antonio Brack über die Tunque-Scheide nach Chuchurras im Palcazu-Tal. Sie durchquerte ein Dutzend verschiedene geologische Bodenschichten, jede mit ihrer eigenen Flora. Es handelte sich um einen Weg, den die deutschen Familien im Palcazu-Tal benutzten, um ihr Vieh (Schweine, glaube ich) zu den Märkten auf der anderen Seite der Berge (d.h. Oxa usw.) zu bringen, bevor es eine Landebahn in Isco (und keine Straße) gab. Ich vermute, dass der Weg jetzt überwachsen und unmöglich zu verfolgen ist, aber vielleicht gibt es neuere Pfade, die von der Parkstation am Rio Isco (Iscosazín) hinaufführen. Es zeigte mir, dass der Yanachaga (Yanachaga Gebirgszug) ein geologischer ,Schichtkuchen' war, der so aufgeschüttet wurde, dass auf der Ostseite alle Schichten freigelegt wurden, aber auf der Westseite geologisch einheitlicher war, mit Ausnahme der Spitzen des Bergrückens." Eine Karte des Nationalparks ist dem Internet zu entnehmen (https://de.wikipedia.org/wiki/ Nationalpark_Yanachaga_Chemillén). 


\section{Der Nationalpark Yanachaga Chemillén}

Der Nationalpark befindet sich in Zentral-Peru und erstreckt sich über die Cordillera Yanachaga, die zum östlichen Bereich der Anden gehört, überschreitet im Norden an der engsten Stelle des Tals des Rio Huancabama denselben und hat dort seine höchste Erhebung (3643 m). Die Landschaft wird von dicht bewaldeten Bergen mit tiefen Schluchten dominiert. Insgesamt ist das Gelände sehr unwegsam mit felsigen und überwiegend steilen, bewaldeten Hängen. Die tiefsten Punkte sind das Tal des Río Huancabamba (später Río Pozuzo) mit $1000 \mathrm{~m}$ im Nordwesten und das Tal des Río Iscosazín im Osten mit $370 \mathrm{~m}$ über dem Meeresspiegel. Mit mehr als $3000 \mathrm{~m}$ Höhenunterschied ist es selbstredend, dass unterschiedliche Klimata anzutreffen sind. In den höchsten Lagen gibt es extreme Feuchte, teils sumpfige von niedrigem Gebüsch und Gräsern bestandene Pajonales (Pampa), darunter Bergregenwald und in den noch tieferen Lagen tropischen Regenwald. Die Niederschläge erreichen $6000 \mathrm{~mm}$ an der Ostflanke (PalcazúFlanke) und $1500 \mathrm{~mm}$ bis $2200 \mathrm{~mm}$ an der Oxapampa-Flanke. Die stark unterschiedlichen
Niederschlagsmengen zusammen mit geografischen Begebenheiten resultieren in einer Vielfalt ökologischer Nischen, die eine enorme Artenvielfalt aufweisen.

\section{Erstes Herantasten an den Nationalpark}

Am 6. Dezember 2019 startete die Exkursion von Oxapampa aus in das unwegsame Gelände des Nationalparks. In einem Schluchtwaldrest auf 2060 m Höhe zeigte sich eine überwältigend üppige Vegetation. Kein Zentimeter Ast der Bäume war frei von Moosen oder epiphytischen Blütenpflanzen, und es gab jede Menge Kleinorchideen, von denen viele gerade in Blüte standen.

Besonders faszinierend waren die kleinen Lepanthes-Arten. Es ist kaum vorstellbar, dass diese wie unsere heimischen Ophrys-Arten Sexualtäuschblumen sein sollen. Getäuscht werden hier allerdings keine solitären Bienen (Hymenopteren), sondern kleine Fliegen (Dipteren). Die farbenprächtigen winzigen Blüten weisen für unser Auge keinerlei Ähnlichkeit mit einem Fliegenweibchen auf. So ist die Anlockung der männ-

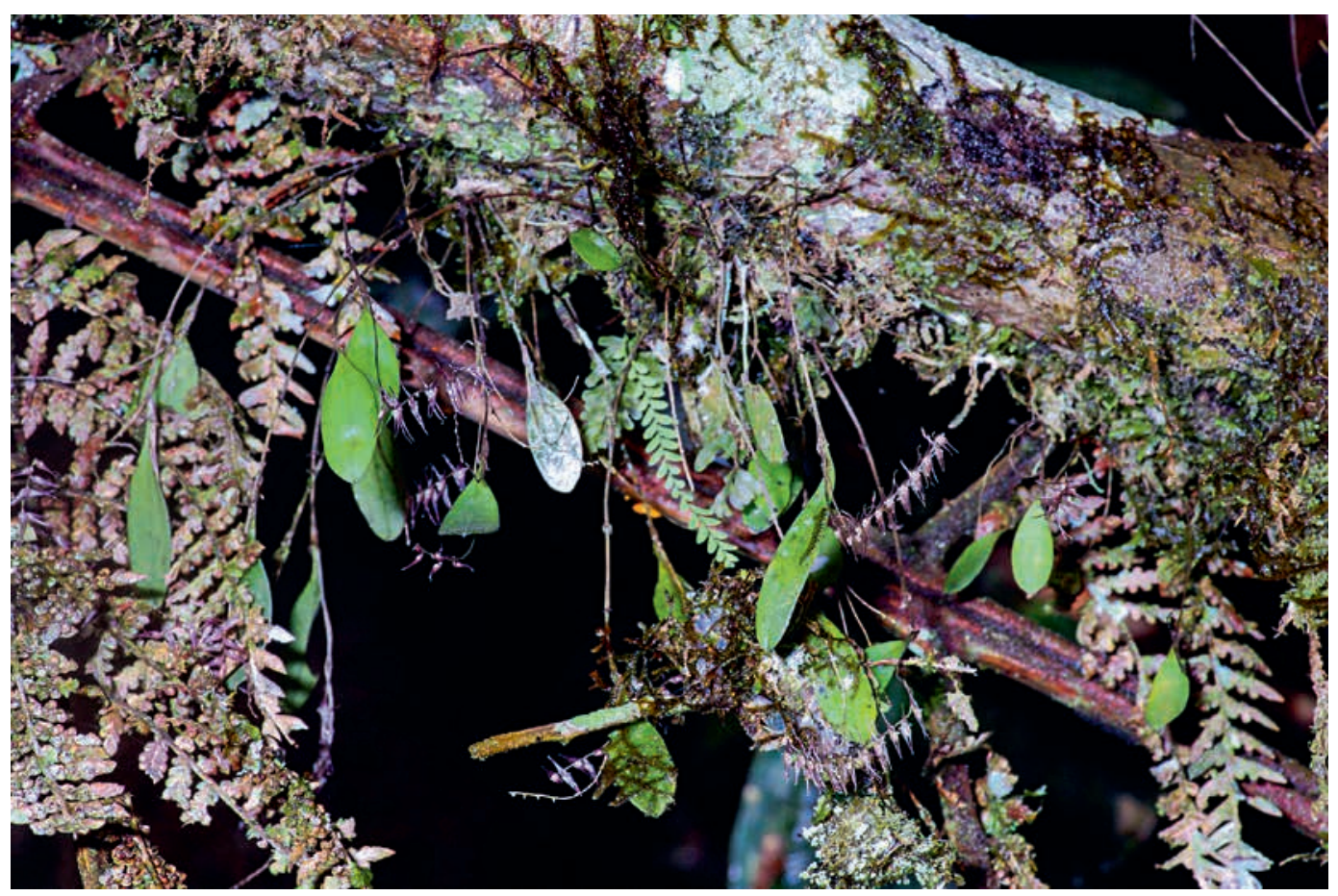

Abb. 1: Mehrere gleichzeitig blühende Lepanthopsis acuminata (Orchidaceae) Pflanzen auf einem Ast. (Foto: G. GerLach) 


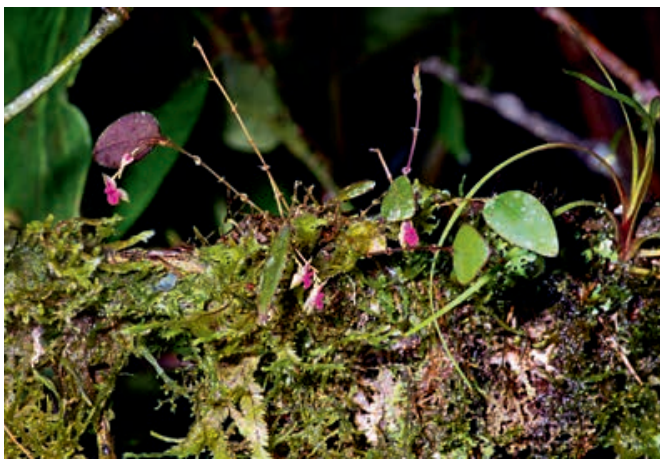

Abb. 2: Ein Mikrokosmos auf einem dünnen Zweig, eine Lepanthes-Art mit $1 \mathrm{~cm}$ langen Blättern (Foto: G. Gerlach)
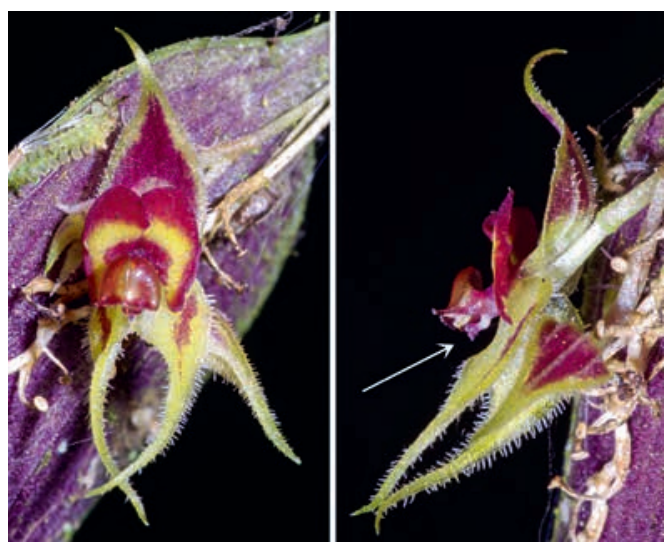

Abb. 3: Lepanthes sp. in Seitenansicht; der Pfeil weist auf das Lippenanhängsel hin, der Andockungstelle für das Genital der kleinen männlichen Diptere. Blütengröße etwa $5 \mathrm{~mm}$. (Foto: G. Gerlach)

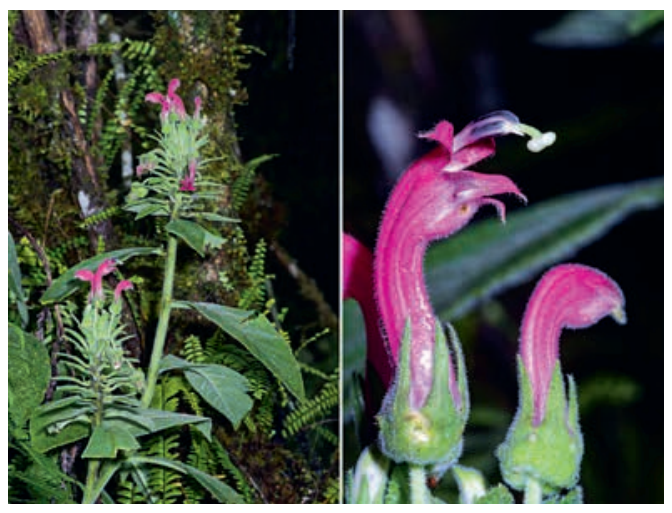

Abb. 4: Centropogon umbrosus (Campanulaceae) wird von Kolibris bestäubt und weist sekundäre Pollenpräsentation auf. Die geöffnete Blüte zeigt ihre zu einer Röhre verwachsenen Staubblätter, durch die sich bereits der Griffel mit der hier aufgeklappten Narbe durchgeschoben hat, die Blüte ist somit in der weiblichen Phase. (Foto:.G. Gerlach) lichen Fliegen nur mit dem Duft zu erklären, der wiederum für uns nicht wahrnehmbar ist. Ist das Fliegenmännchen auf der Blüte gelandet, verhakt es sich mit seinen Genitalien in einen Anhang der Orchideenlippe und gibt gar in manchen Fällen sein Sperma ab. Die Orchideenblüte besteht aus den Sepalen (äußerer Blütenkreis), den Petalen, der Lippe (innerer Blütenkreis) und der Säule, dem Verwachsungsprodukt der reproduktiven Organe. Die Funktion der Lippe ist es, den Bestäuber so zu positionieren, dass konstant an derselben Stelle des Körpers das Pollinarium (der gesamte Pollen) fixiert wird. So führen taktile Reize zusammen mit dem arttypischen Duft zur Täuschung des Insekts und zur Bestäubung der Blüte.

In dem Lebensraum des Bergregenwaldes dürfen natürlich nicht die von Kolibris bestäubten Pflanzen fehlen, die mit leuchtenden Farben protzen. Vogelblüten locken ihre Bestäuber mit auffälligen Farben an, dabei spielen vor allem Rottöne eine wichtige Rolle. Neben Fuchsia abrupta (Onagraceae) fällt das staudig wachsende Centropogon umbrosus (Campanulaceae, früher Lobeliaceae) auf. Wie alle Glockenblumengewächse und die nahe verwandten Körbchenblütler (Asteraceae) zeigt diese Verwandtschaftsgruppe sekundäre Pollenpräsentation. Dabei wird der Pollen nicht von den Staubblättern auf dem Bestäuber abgeladen, sondern durch Vermittlung eines Zwischenlagers. Die Staubblätter dieser Pflanzengruppe sind zu einer Röhre verwachsen, sie öffnen sich nach innen. Durch diesen Hohlzylinder wächst der Griffel, den Pollen vor sich herschiebend. Die Narbe ist in diesem Stadium noch geschlossen. So entsteht zunächst am Ende der Röhre ein Pollenhäufchen, das dem Bestäuber appliziert wird. Mit weiterem Wachstum des Griffels öffnet sich die Narbe, die fertilen Narbenlappen krümmen sich nach hinten. Selbstbestäubung wird vermieden, da die empfängnisfähigen Narbenlappen nicht in Kontakt mit dem eigenen Pollen kommen. Sie sind geschützt, da sie zusammengeklappt nach innen liegen. Durch sekundäre Pollenpräsentation wird eine hohe Präzision bei der Pollenübertragung erreicht. Die Narbe tritt später an genau dieselbe Position, die vorher das Pollenhäufchen eingenommen hatte. Nicht 


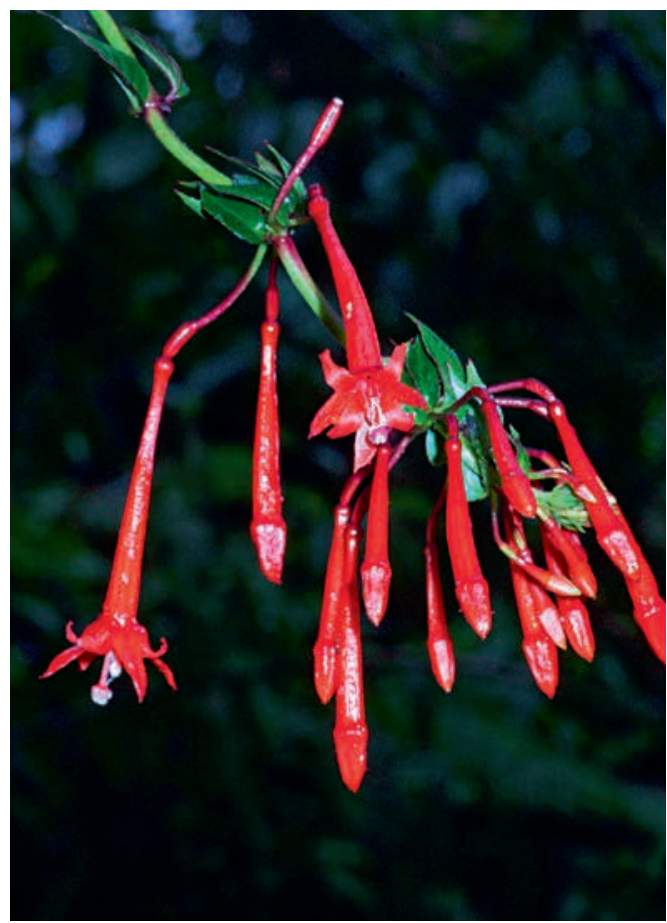

Abb. 5: Fuchsia abrupta (Onagraceae) ist eine typische Kolibriblüte mit engen Blütenröhren. (Foto: G. GerLach)

nur bei den beiden genannten Pflanzenfamilien tritt die sekundäre Pollenpräsentation auf, ihre unterschiedlichen Mechanismen im Pflanzenreich sind mannigfaltig. Dieses Phänomen wird bei den dikotylen sowie den monokotylen Pflanzen angetroffen. Es entstand im Laufe der Evolution der Blütenpflanzen unabhängig an verschiedensten Positionen im Pflanzensystem.

Nicht nur die Viehwirtschaft, sondern auch die Kultur von Granadillas führen hier in der Gegend um Oxapampa zum Rückgang des Regenwaldes. Passiflora ligularis (Passifloraceae) ist einer der Exportschlager Perus, die orangefarbigen Früchte werden häufig in Deutschland angeboten. Wegen ihrer harten Schale sind sie gut zu transportieren, also ideal, um sie weiträumiger zu vermarkten. Der Nachteil dieser Monokulturen liegt klar auf der Hand, sie ist spritzintensiv und zerstört Lebensraum von Pflanzen und Tieren. In manchen Bereichen der Provinz Oxapampa sind ganze Hänge von den berankten Gestellen überzogen. Unter den Granadillas wächst nichts, denn es werden

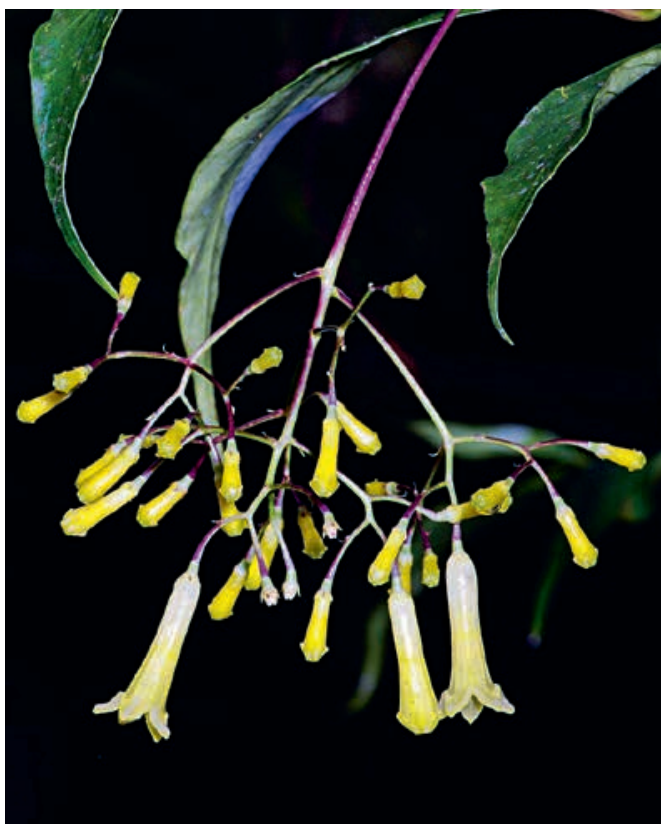

Abb. 6: Die zu 4 cm langen Röhren verwachsenen Blütenblätter der Palicourea calantha (Rubiaceae) verbergen den Nektar für ungebetene Blütenbesucher. Nur Kolibris mit entsprechend langem Schnabel vermögen ihn auszubeuten. (Foto: G. Gerlach)

massiv Herbizide eingesetzt. Erosion ist eine weitere negative Folge dieser Kultur, da sie auch an sehr steilen Hängen angelegt wird.

\section{Im Nationalpark, Paujil und seine Umgebung}

Der Nationalpark Yanachaga Chemillén ist nur an wenigen Stellen erreichbar. Obwohl vom Standort Oxapampa ein direkter und schneller Zugang zur Station San Alberto bestand, war es reizvoll, mit der am schwierigsten erreichbaren Station Paujil zu beginnen. Zusammen mit dem Berliner Kollegen Nils Köster wurde die Tour durchgeführt. Paujil liegt an der Basis der Palcazú-Flanke, also jenem Teil des Nationalparks, der wesentlich höhere Niederschläge aufzuweisen hat als die Seite von Oxapampa. Obwohl nur etwa $30 \mathrm{~km}$ Luftlinie vom Quartier in Oxapampa entfernt, auf der anderen Seite der Yanachaga Kordillere, ist es sehr mühsam, dorthin zu gelangen. Die Fahrt geht mit dem Auto über Villa Rica in das Tal des Río Palcazú. Von dort fährt man dann von der Ansiedlung Iscosazín den Rio 


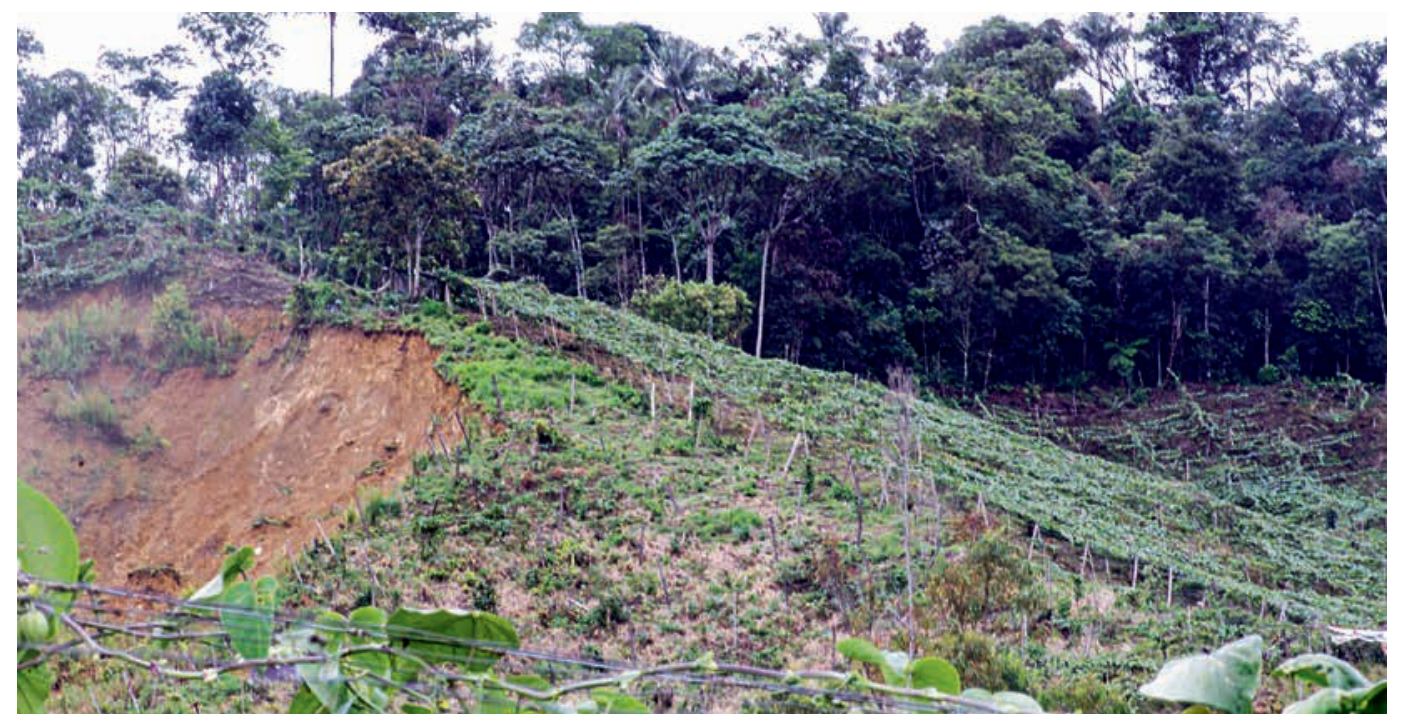

Abb. 7: Granadilla-Feld am Rand des Regenwaldes. Gut ist auch die einsetzende Erosion zu sehen, begünstigt durch die offene Bodenkrume. (Foto: G. Gerlach)
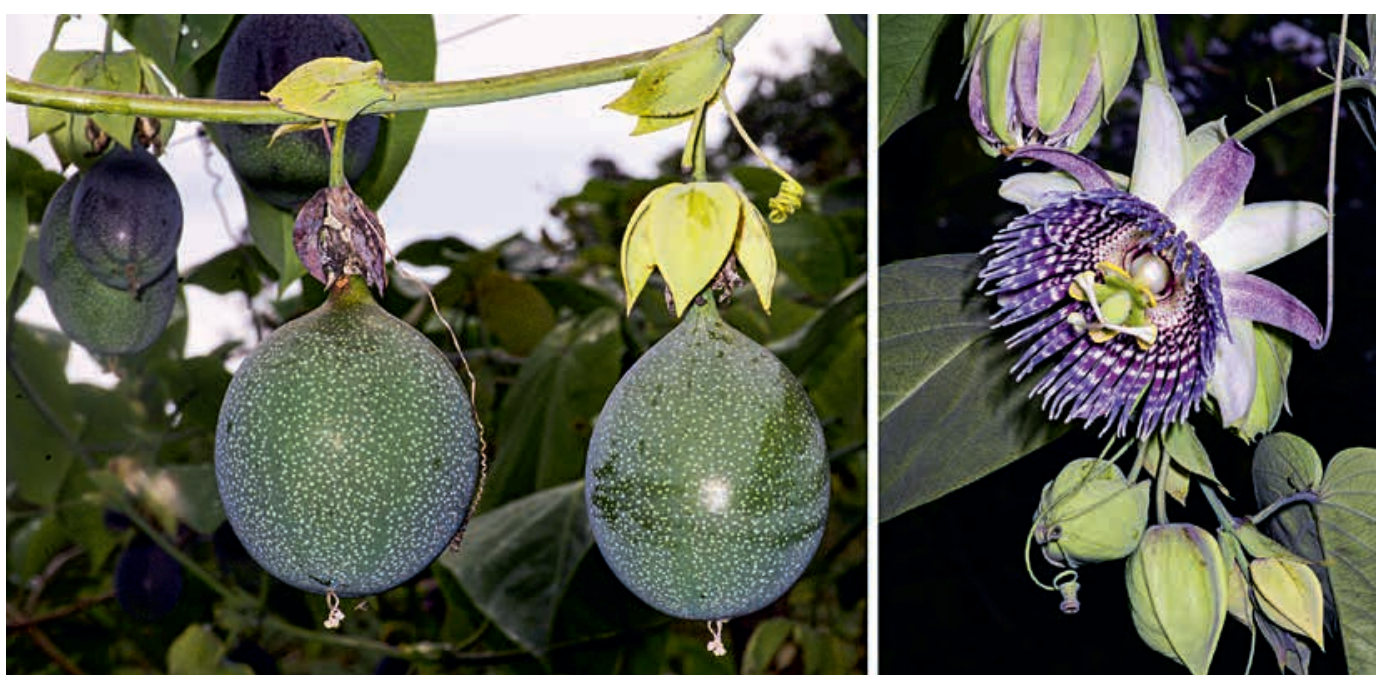

Abb. 8: Die Granadilla Passiflora ligularis (Passifloraceae) wird an Drähten gezogen. Die grünen Früchte sind noch unreif, sie färben sich später orangefarben. (Foto: G. GERLACH)

Iscosazín aufwärts. Dieser Fluss ist bekannt für seine Stromschnellen und es gibt nur sehr wenige Bootsführer, die sich trauen, ihn zu befahren. Paujil liegt auf $370 \mathrm{~m}$ über dem Meeresspiegel, dort herrscht also tropisches Tieflandklima. Die Station liegt an der Mündung des Río Paujil in den Río Iscosazín, von dort ausgehend lassen sich die Berge oder der Uferwald erkunden. Direkt am Waldrand um die Station finden sich die ersten spektakulären Pflanzen wie Microgramma bifrons (Polypodiaceae) ein epiphytischer Farn, der Myrmekodomatien (myrmex - Ameise; domus - Haus) besitzt. Die Ameisen besetzen die aufgeschwollenen hohlen Sprossteile an der Basis der Blätter, sie verteidigen vehement ihre Behausung, somit ihre Pflanze. Solche AmeisenPflanzen-Beziehungen sind in den Tropen häufig zu finden, auch hier wieder in den unterschied- 


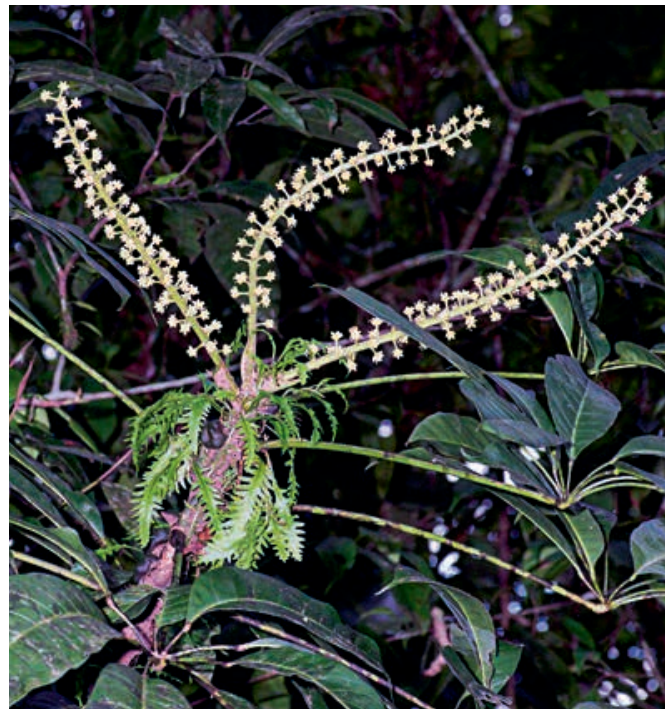

Abb. 9: Der epiphytische Farn Microgramma bifrons (Polypodiaceae) wächst auf einer Schefflera sp. (Araliaceae).

(Foto: G. Gerlach)

lichsten Pflanzenfamilien und verteilt über das gesamte Pflanzenreich.

Unweit der Station im schattig-feuchten Unterwuchs fand sich ein weiterer interessanter Farn, nämlich Schizaea elegans (Schizaeaceae). Als Jungpflanze ist er kryptisch bräunlich gefärbt, um so vielleicht von potenziellen Fraßfeinden nicht erkannt zu werden. Bei den Jungpflanzen sind die Wedelränder noch ganzrandig und eingerollt. Im Laufe der weiteren Entwicklung differenzieren sie sich, an den Enden der Wedelnerven entstehen die Sori, in denen sich die Farnsporen entwickeln.

Ein weiterer Vertreter der Farne wächst als mächtige Kletterpflanze. Es handelt sich um Salpichlaena volubilis ssp. amazonica (Blechnaceae). Dieser Farn windet und klettert mit dem Stiel des Wedels der gefiederten Blätter, die Wedel erreichen mehrere Meter Länge. Im fertilen Stadium entwickelt sich das schützende, pergamentartige Indusium entlang der Mittelrippe der Blättchen und rollt sich schließlich ein, um dann die Sporen freizugeben

Der Urwald ist von hohen Bäumen dominiert, teils ist er aber auch niedriger. Auffällig ist der

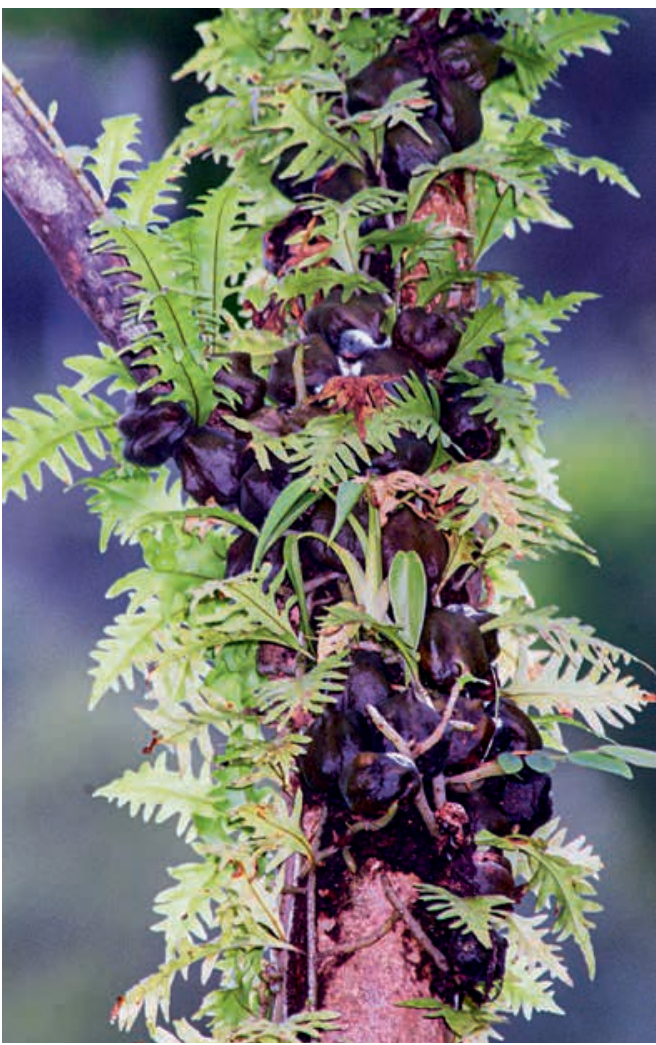

Abb. 10: Der von Ameisen besiedelte, epiphytische Farn Microgramma bifrons mit seinen knolligen, dunklen Myrmekodomatien. (Foto: G. Gerlach)

Epiphytenreichtum. Sehr viele Orchideen waren gerade in Blüte, im Januar gab es viele MaxillariaArten, dazu Scaphyglottis und die größtblütigste Octomeria-Art, die dem Autor jemals zu Gesicht gekommen ist.

Wie überall in den neuweltlichen Tropenwäldern stechen sofort die farbenprächtigen, von Kolibris bestäubten Blüten ins Auge. Diesmal war es der Halbparasit Psittacanthus zonatus (Loranthaceae), der mit seinen kontrastreich rot/schwarzpurpurn gebänderten Blüten um Augenmerk heischte. Die Blütenblätter sind zu ca. $8 \mathrm{~cm}$ langen, engen leuchtend roten Röhren verwachsen, die am Schlund nochmals zusammengezogen und mit einem dunklen Band versehen sind. Als Bestäuber kommen hier nur langschnäbelige Kolibris in Betracht, die in Genuss des tief verborgenen Nektars kommen können. 


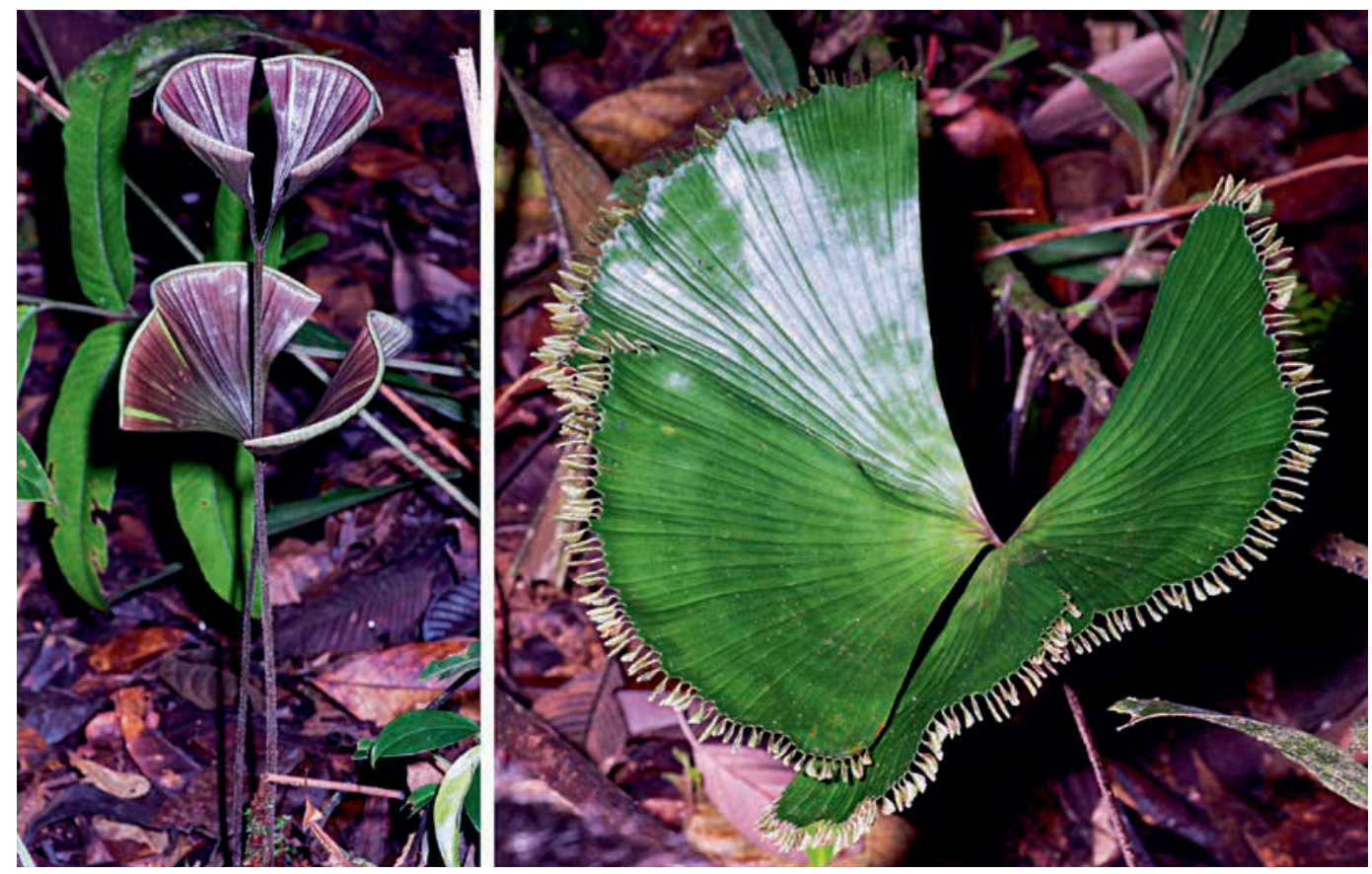

Abb.11: Schizaea elegans (Schizaeaceae), links noch kryptisch gefärbte Jungpflanze, rechts nahezu fertile Pflanze mit den Sori an den Enden der Wedeladern. (Foto: G. Gerlach)
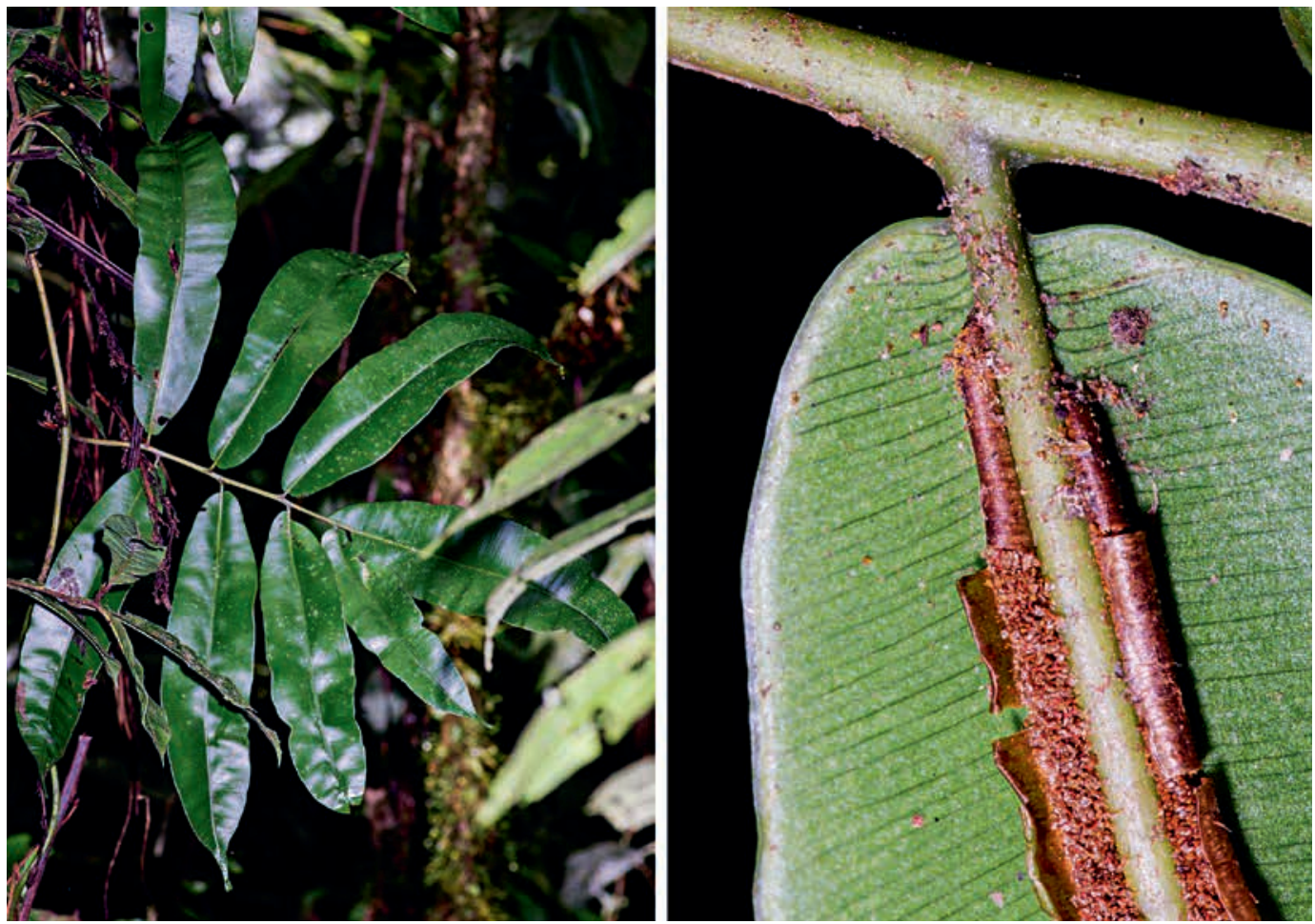

Abb. 12: Salpichlaena volubilis ssp. amazonica (Blechnaceae) mit meterlangen windenden Wedeln. (Foto: G. GerLACH) 

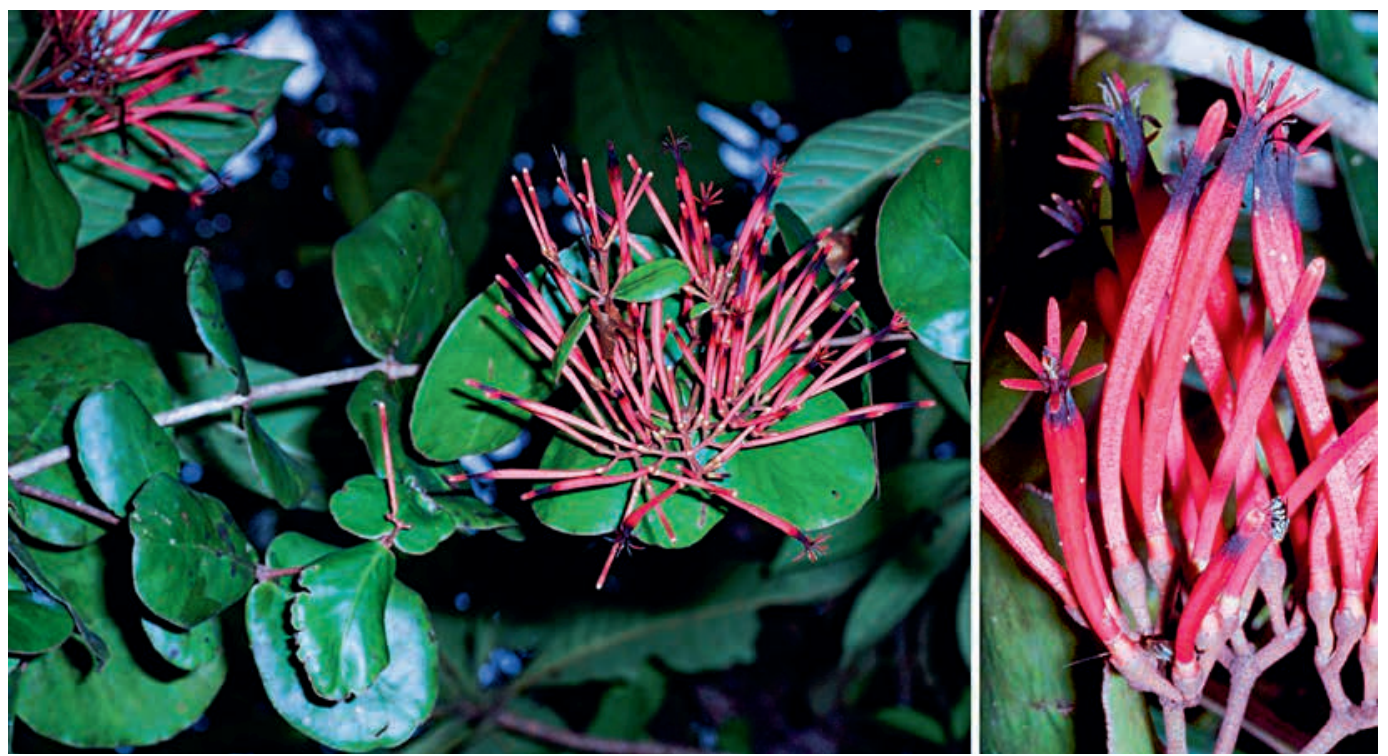

Abb. 13: Der Halbparasit Psittacanthus zonatus (Loranthaceae). (Foto: G. GerLACH)

Viele Gesneriengewächse werden als Zimmerpflanzen sehr geschätzt, denn sie bilden meist farbige Blüten, vor allem in den attraktiven Rottönen. Es handelt sich meist um krautige Pflanzen, manche sind aber auch verholzt. In der Natur finden sie sich zumeist in schattigen Bereichen des Unterwuchses. Es gibt terrestrisch wachsende, epiphytische und lianenartige unter ihnen. Bei der Mehrzahl der Arten sind die Blütenblätter zu einer Röhre verwachsen, am Grunde der Blütenröhre befinden sich Drüsen, die Nektar absondern. Es gibt eine Reihe von verschiedenen Anpassungen an die Bestäuber, so gibt es die auffällig zumeist rotblütigen Arten, die von Vögeln, in der Neotropis also von Kolibris, bestäubt werden. Es gibt weiße Blüten mit engen langen Röhren, die von Schwärmern nachts bestäubt werden, blaue (z.B. Saintpaulia-Arten, Usambaraveilchen) die Bienenblüten sein dürften, und es gibt sogar welche mit grünen oder grünbraunen Blüten, die von Blumen-Fledermäusen bestäubt werden. Im Folgenden werden einige kolibriblütige Vertreter aus dieser Pflanzenfamilie vorgestellt.

Die kletternde Drymonia semicordata bildet gelbe Blüten, die in auffälligem Kontrast mit den roten Kelchblättern stehen. Hier haben die leuchtend roten Kelchblätter Teil an der Anlockung der

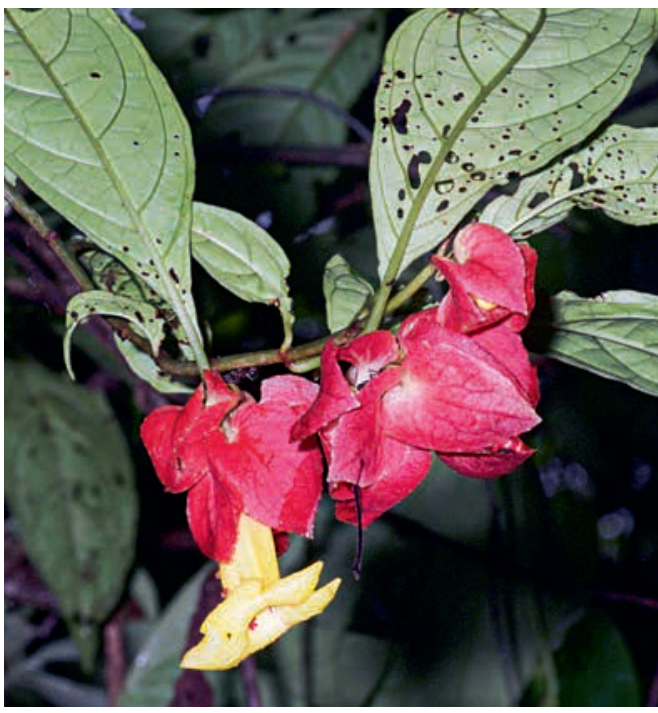

Abb. 14: Die kletternde Drymonia semicordata (Gesneriaceae) besitzt gelbe Blüten, die in auffälligem Kontrast mit den roten Kelchblättern stehen (Foto: G. Gerlach)

Vögel. Die Blüten hängen nach unten, so ist der Nektar gut gegen Verdünnung durch den reichlichen Regen im Urwald geschützt. Zudem können sie nur von den Kolibris ausgebeutet werden, die den Schwirrflug beherrschen und so, vor der Blüte stehend, mit ihren langen Schnäbeln und Zungen an den begehrten Nektar gelangen. 


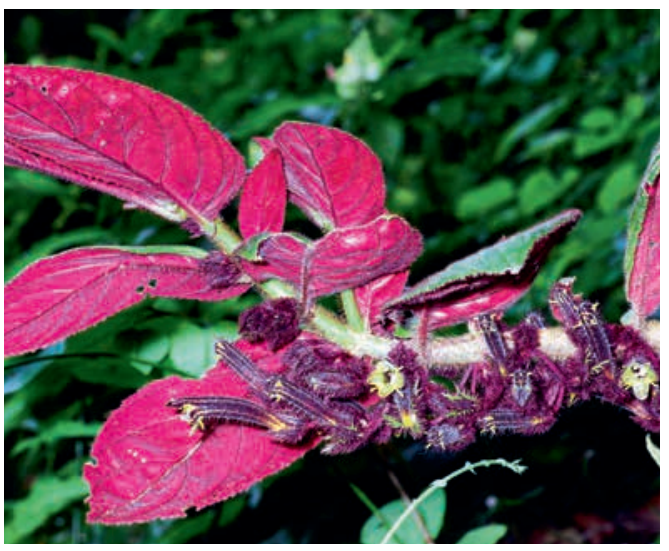

Abb. 15: Columnea purpureovittata (Gesneriaceae) mit prächtiger Blattunterseite. (Foto: G. Gerlach)

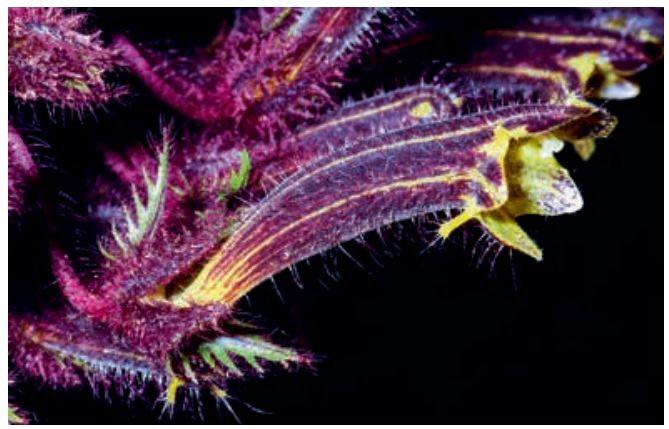

Abb. 16: Columnea purpureovittata (Gesneriaceae) in der weiblichen Phase, die Narbenlappen sind geöffnet (Foto: G. Gerlach)
Ganz anders ist die Situation bei Columnea purpureovittata. Hier sind die Blüten nicht besonders farbenprächtig, sie sind purpurbraun und weisen gelbe Streifen auf. Der Kelch ist ebenso gefärbt, kontrastiert also nicht zur Blüte. Betrachtet man aber die Pflanze von der Unterseite, so ist festzustellen, dass die Blätter unterseits wunderschön rot gefärbt sind. Sie übernehmen hier die Anlockungsfunktion für die Kolibris. Gesneriaceen haben meist vormännliche Blüten. Es entwickeln sich zunächst die Staubblätter und die Narbe befindet sich noch tief in der Blütenröhre versteckt. Nachdem der Pollen präsentiert wurde, rollen sich die Staubfäden ein und der Griffel streckt sich, so dass die Narbe dieselbe Höhe in der Blütenröhre einnimmt, die zuvor die Staubblätter hatten. Dann öffnet sich die Narbe mit ihren zwei Narbenlappen (Abb. 16), die Blüte befindet sich nun in der weiblichen Phase.

Ganz ähnlich ist die Anlockungsfunktion bei Columnea guttata. Die Blattspitzen sind unterseits blutrot gefärbt und bilden einen auffälligen Kontrast zu der restlichen silbrig-grünen Blattspreite. Im Gegenlicht leuchten die roten Bereiche ähnlich wie Kirchenfenster, sie werben um Aufmerksamkeit, locken den vorbeifliegenden Kolibri zu sich hin. Die Blüten sind zwar leuchtend gelb gefärbt,
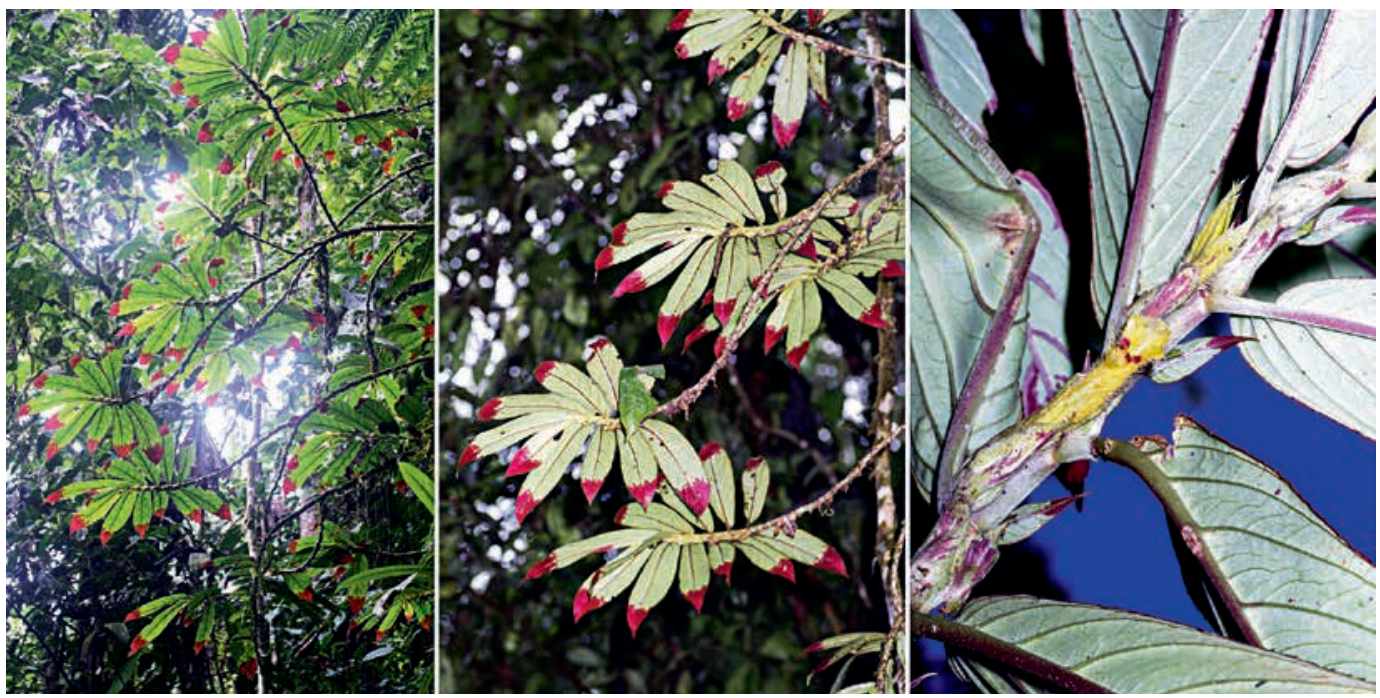

Abb. 17: Wie von außen beleuchtete Kirchenfenster sehen die Blätter von Columnea guttata (Gesneriaceae) im Gegenlicht aus. (Foto: G. Gerlach) 

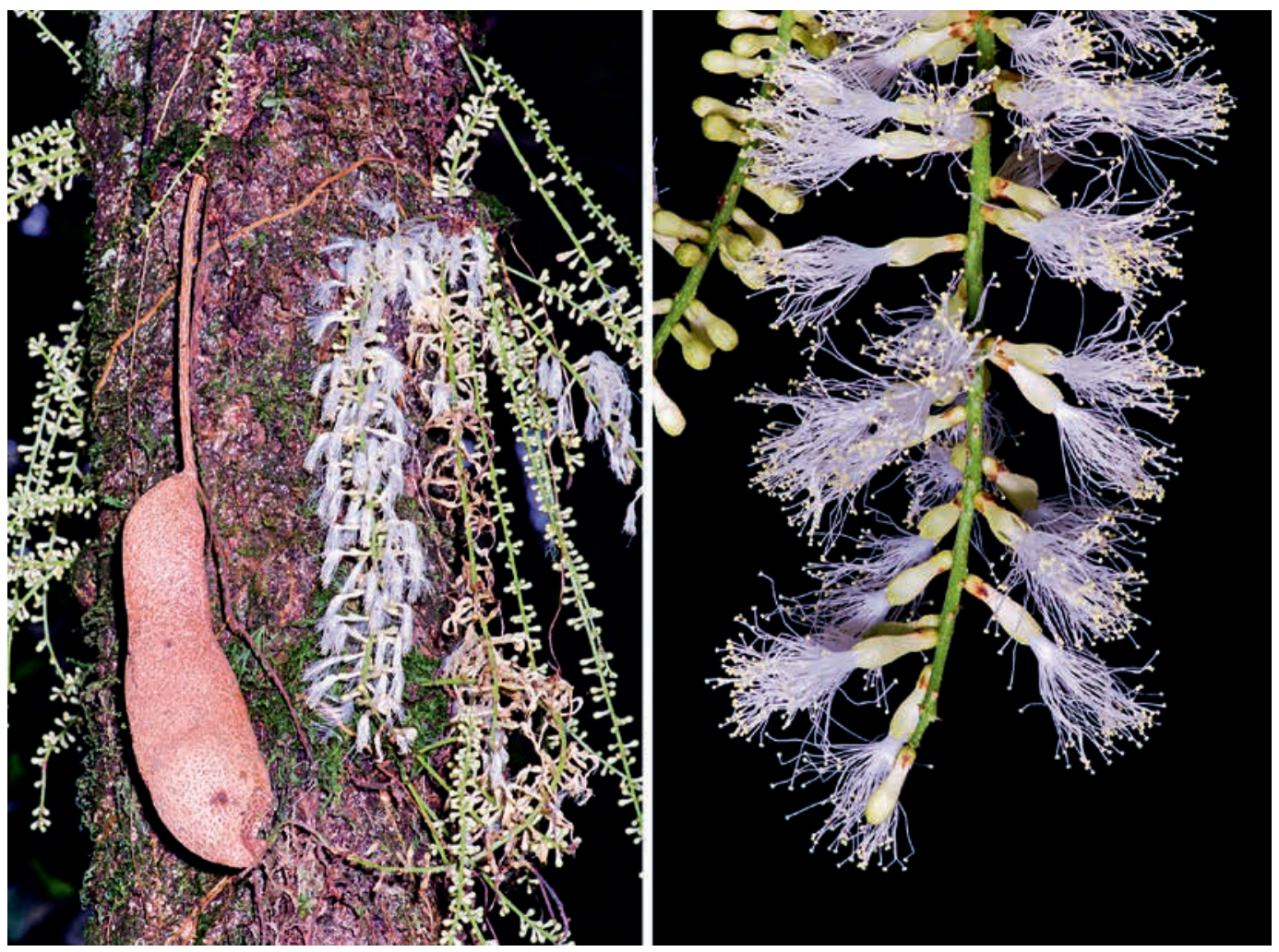

Abb. 18: Zygia sp. (Fabaceae) ist stammblütig, cauliflor. Die Blüten öffnen sich nachts, bei der linken Pflanze sind sie schon welk, da das Foto tagsüber aufgenommen wurde. Beim rechten Blütenstand stehen sie noch voll im Saft. (Foto: G. GerLACH)

fallen aber kaum in der Entfernung auf. In der Nähe aber werden sie leicht vom Bestäuber gefunden. Viele der Kolibri-Arten sind sogenannte „Trapliner". Sie verhalten sich wie ein Trapper, der seine gestellten Fallen aufsucht. Er folgt einer ihm bekannten Route und findet so alle seine Fallen. Ähnlich verhält sich der Vogel. Er kennt seine blühenden Pflanzen und sucht eine nach der anderen auf. Da diese Blüten kontinuierlich Nektar produzieren, besucht er sie mehrfach am Tag. Für die Blüte ist es wichtig, dem Bestäuber nicht zu viel anzubieten, denn sonst würde er einfach verweilen und nicht zur nächsten Blüte eilen. In der Evolution hat sich die produzierte Nektarmenge so fein eingestellt, dass der Bestäuber gerade so auf seine Kosten kommt. Der Schwirrflug des Kolibris ist engergieaufwendig, der Vogel bekommt also etwas mehr Nektar, als er für seine An- und Abreise benötigt. Columnea guttata und einige weitere Vertreter der Gattung trainieren ihre Bestäuber, denn auch wenn keine Blüten vorhanden sind, leuchten die Blattspitzen wie Kirchenfenster. Der Kolibri behält die Pflanze in seiner Route, schaut kurz nach, ob es etwas zu holen gibt und fliegt weiter, wenn keine Blüte zu sehen ist. Das Verhalten der Vögel an den Blüten kann sich der erfahrene Fotograf zu Nutze machen. Die Tiere sind pünktlich in ihrem Fahrplan. Stoppt man die Zeit zwischen den Blütenbesuchen, so lässt sich der Nächste berechnen und so kommt der Fotograf eventuell zum erfolgreichen Schuss.

Im Gegensatz zu den tagblütigen Pflanzen zeigen nachtblütige keine leuchtenden Farben, sondern sind reinweiß im Falle von Nachtfalterblumen oder schmutzig grün-bräunlich im Falle von Fledermausblumen. Zygia sp. (Fabaceae) wird mit großer Wahrscheinlichkeit von Nachtfaltern, vermutlich Schwärmern (Sphingidae), bestäubt. Die Blütenblätter der Art sind unscheinbar, der 

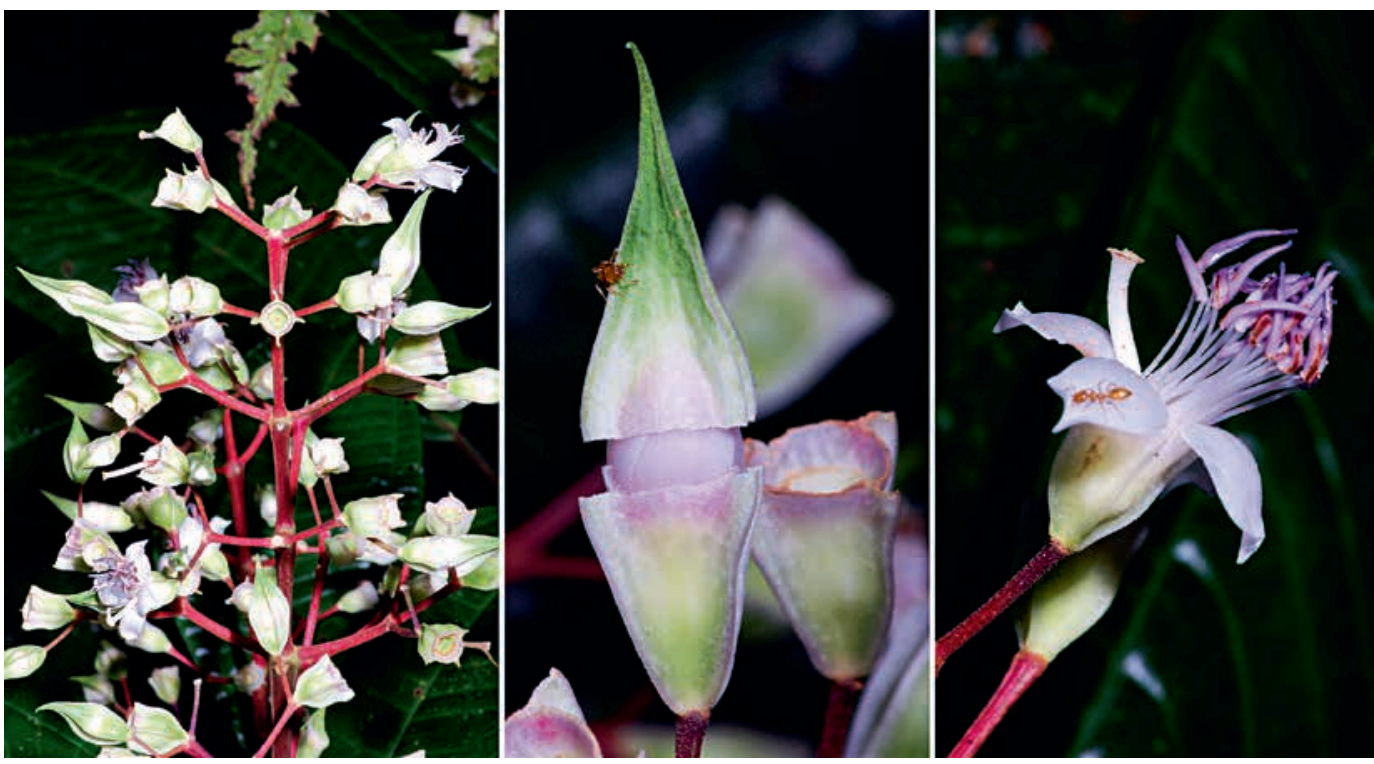

Abb. 19: Miconia valenzuelana (Melastomataceae), besitzt ein Trennungsgewebe in der Mitte der Kelchblätter und stößt beim Erblühen die obere Hälfte des Kelches als Kalyptra ab. (Foto: G. Gerlach)

Schauapparat wird allein von den zahlreichen langen Staubblättern gebildet, die basal zu einer Röhre verwachsen sind und in der Blütenblattröhre steckt.

Beim Durchstreifen des Urwaldes entlang des Flusses fiel ein seltsames Schwarzmundgewächs (Melastomtaceae) auf, dessen Blütenknospen besonders schlank und spitz waren. Die Spitzen fanden sich nicht an den geöffneten Blüten, denn sie waren bereits abgefallen. Bei der Mehrzahl der Blüten haben die Kelchblätter u.a. die Funktion des Schutzes der Blütenblätter während der Blütenentwicklung. Oft sind die Kelchblätter auch derber als die meist zarten Blütenblätter, so dass sie einen effektiven Schutz darstellen. Bei einigen Pflanzen werden die Kelchblätter ganz oder teilweise beim Erblühen abgestoßen. Bei der angetroffenen Miconia valenzuelana findet sich ein Trennungsgewebe quer zur Längsachse in den bei der Art verwachsenen Kelchblättern. Beim Fortschreiten der Blütenentwicklung reißt die Spitze $\mathrm{ab}$, sie wird abgesprengt und als Mütze (Kalyptra) abgestoßen. Bei der Art ist diese Mütze besonders auffällig, die Dehiszenz der Kelchblätter aber ist bei Schwarzmundgewächsen ein häufiges Phänomen. Eine ähnliche Kalyptra wird bei der
Gattung Marcgravia (Marcgraviaceae) angetroffen, dort werden allerdings die verwachsenen Blütenblätter als Mütze abgestoßen.

Die faszinierende Exkursion ging zu Ende mit einer abenteuerlichen Bootsfahrt auf dem Rückweg und dem Befall von parasitischen Milben mit zwei Wochen anhaltendem Juckreiz. Als Botaniker nimmt man dies aber in Kauf, da es so faszinierend ist, tropische Pflanzen nicht nur in Gewächshäusern Botanischer Gärten betrachten zu können, sondern sie auch in ihrem natürlichen tropischen Lebensraum mitsamt ihrer Bestäuber beobachten zu können.

\section{Anschrift des Autors}

Dr. Günter Gerlach, Botanischer Garten MünchenNymphenburg, Staatliche Naturwissenschaftliche Sammlungen Bayerns (SNSB), Menzinger Str. 65, 80638 München, E-Mail: gerlach@snsb.de;

https://orcid.org/0000-0002-2819-7218

Gastwissenschaftler: Jardín Botánico de Missouri, Prolg. Bolognesi Mz. E, Lote-6, Oxapampa, Peru

Gastwissenschaftler: Museo de Historia Natural, Universidad Nacional Mayor de San Marcos, Av. Arenales 1256. Jesús Maria, Lima, Peru 